\title{
ORIGINAL ARTICLE \\ Rewards of parenting children and adolescents with spinal cord injuries
}

\author{
M Morrison ${ }^{1}$, EH Kelly ${ }^{2,3}$, HF Russell ${ }^{4}$ and LC Vogel $^{2,5}$
}

Study design: Mixed-method study.

Objectives: Describe caregiver perspectives on the rewards of parenting youth with spinal cord injury (SCI) and explore the relationships between rewards and child/caregiver demographic characteristics and child psychosocial outcomes.

Setting: Data collection occurred at three pediatric specialty hospitals within a single hospital system in the United States.

Methods: Self-identified primary caregivers $(n=178)$ of children aged $1-18$ years answered the question: 'What has been most rewarding in parenting a child with SCl'? and completed a questionnaire about their child's health-related quality of life (HRQOL). Participants aged $7-18$ years $(n=134)$ also completed tools assessing their community participation, anxiety, depression and HRQOL. Results: Four reward themes emerged: Enhanced Resilience (for example, resilience in my child, self and family), Caregiver-Child Relationship, Connecting with Others, and Learning. Caregivers of children with lower self-reported school and overall psychosocial HRQOL were more likely to report Enhanced Resilience in their child. Caregivers whose children had fewer depressive symptoms, lower levels of participation and who were older at injury and interview felt rewarded by an enhanced Caregiver-Child Relationship. Caregivers of children with a broader context of participation and higher school and psychosocial HRQOL reported Connecting with Others. Finally, unemployed caregivers and those with less education were more likely to report Learning.

Conclusions: Caregivers reported a variety of rewards from parenting their children with SCl, and several relationships emerged between rewards and demographics and child psychosocial outcomes. Future research should further examine the positive experiences of caregivers and whether focusing on strengths might yield better long-term outcomes for children with SCl.

Spinal Cord (2017) 55, 52-58; doi:10.1038/sc.2016.83; published online 21 June 2016

\section{INTRODUCTION}

It is estimated that in the United States 5.9 million children with severe physical injuries live at home and their parents or other family members often become their caregivers. ${ }^{1}$ One subgroup of this larger category includes children and adolescents with spinal cord injuries (SCIs). These youth may experience physical complications including paralysis, incontinence and pain as well as psychological difficulties. ${ }^{2,3}$ After injury, children undergo acute rehabilitation to develop skills necessary to maintain physical and emotional health, incorporate these skills into daily functioning and reintegrate back into the community. ${ }^{4-6}$ During this period and thereafter, caregivers (that is, typically parents) are critical to a child's life, providing youth with the medical, physical and emotional resources necessary for adjustment and development. ${ }^{7-9}$

Many past studies have focused on the challenges caregivers face in managing their child's chronic condition, ${ }^{10,11}$ but few have investigated the rewards caregivers might derive from this experience. Highlighting and fostering a resilience approach may have tremendous positive implications that can impact the caregiver. In particular, taking a positive, strength-based approach broadens the examination of any critical event by giving equal attention to an individual's strengths, hopes and rewards. This can provide for a more comprehensive-and accurate- understanding of the complexity of an event, helping to make sense of the human experience. Incorporating a strength-based approach may serve to enhance resilience factors and can provide deeper insight into the complex role of being a caregiver.

Indeed, past research has found resilience to be a protective factor against negative affect ${ }^{12}$ and caregiver burden. ${ }^{13}$ Caregiver's physical health may also be positively affected by resilience. In a study that examined caregivers of children with autism spectrum disorder, the authors found that highly resilient caregivers had better perceived health, higher social support and lower symptoms of stress as reflected in lower levels of morning cortisol levels. ${ }^{14}$ So what is unique about these resilient caregivers? Past research indicates that resilient caregivers employ a variety of skills to meet difficult challenges. In a study among caregiving family members of people with traumatic brain or SCI, caregivers who were deemed as resilient utilized a wider range of strategies to meet the challenges of caregiving and displayed more positive affect. ${ }^{15}$ This is critical in light of research finding that how a caregiver responds to adverse events can influence their adaptive functioning and well-being. In particular, in a study on mothers of adolescents with intellectual disabilities, several coping strategies helped reduce the impact of maternal depression symptoms and

${ }^{1}$ Department of Research, Shriners Hospitals for Children, Philadelphia, PA, USA; ${ }^{2}$ Department of Spinal Cord Injury Research, Shriners Hospitals for Children, Chicago, IL, USA; ${ }^{3}$ American Academy of Pediatrics, Elk Grove Village, IL, USA; ${ }^{4}$ Department of Psychology, Shriners Hospitals for Children, Philadelphia, PA, USA and ${ }^{5}$ Department of Pediatrics, Rush Medical College, Chicago, IL, USA

Correspondence: Dr EH Kelly, American Academy of Pediatrics, 141 Northwest Point Boulevard, Elk Grove Village, IL 60007, USA.

E-mail: ekelly@aap.org

Received 22 September 2015; revised 21 March 2016; accepted 29 March 2016; published online 21 June 2016 
feelings of parenting efficacy. ${ }^{16}$ Attending to positive caregiving experiences may offer insight into potential strategies that could be used by other caregivers, regardless of their child's level of ability.

Given that children with disabilities are particularly dependent on their parents for support, particular attention has been paid to the relationship between caregiver experiences and the well-being of those for whom they care. ${ }^{17,18}$ However, as mentioned above, the vast majority of this work has focused on the negative experiences of these caregivers, and the toll that caregiving takes on their well-being. However, similar to parenting any child, in addition to the stressors, it is likely that parents also find rewards in parenting their child with SCI. Although acquiring an SCI is an emotional time for all involved, exploring caregivers' perspectives on the rewards they experience may highlight important protective factors related to caregiver and child psychological health. Yet, to date, no study has examined the rewards that caregivers of youth with SCI might derive from this experience nor has past research investigated relationships between positive caregiving experiences and child psychosocial outcomes. Identifying these rewards may help in the construction of more well-rounded training and support for caregivers as they prepare for and continue in the role of caring for their child.

The purpose of this mixed-methods study was to investigate how caregivers characterize the rewards of parenting their child with SCI, with the intent of complimenting the existing caregiver literature by creating a more comprehensive understanding of the caregiver experience. We further sought to examine the relationship between these rewards and child and caregiver demographic characteristics and child psychosocial outcomes. Integrating positive experiences into the support of caregivers may help ameliorate the stressful demands of caregiving, improve the overall outcomes for children with SCI and help develop more effective training for caregivers as they prepare for and continue in the role of caring for their child. To our knowledge, this is the first study to take a positive, strength-based approach to examining the experiences of caregivers of youth with SCI.

\section{MATERIALS AND METHODS}

\section{Participant recruitment and study procedures}

Participants were part of a larger project that examined relationships between social participation, quality of life (QOL) and psychosocial outcomes among children and adolescents with SCI and their caregivers. English and Spanishspeaking youth with SCI aged 1-18 years who had been injured for at least 1 year and their caregivers were approached during the course of standard medical care for recruitment from one of the three pediatric specialty hospitals within a large single hospital system in the United States. Each of the participating sites secured approval from their respective institutional review board, and all regulatory requirements for research involving human subjects were met. All participants were engaged in the process of informed consent and completed appropriate consent and (if appropriate) assent documentation. Participants then completed questionnaires online or on paper, depending on their preference. They completed questionnaires on their own or were provided with assistance from the researchers if needed (for example, assistance reading items or writing answers).

\section{Instruments}

Demographic and injury-related information. Caregivers filled out a questionnaire regarding their relationship with their child, their child's race and their own sex, race, marital status, education and community type (for example, urban or rural). Caregivers also responded to an open-ended question, 'What has been most rewarding in parenting a child with SCI?' Patient information on sex, level of injury and injury severity were obtained from a medical chart review. The International Standards for Neurological Classification of Spinal Cord Injury was used to assess the injury level and severity. ${ }^{19}$
Youth psychosocial outcomes. Several standardized tools were completed by youth aged 7-18 years to assess their psychosocial functioning. The Revised Children's Manifest Anxiety Survey (RCMAS-2-SF) is a 10-item short-form self-report measure designed to assess the level and nature of anxiety. ${ }^{20}$ Good internal consistency reliability was shown for the current sample (Cronbach's alpha $(\alpha)=0.811)$. The Children's Depression Inventory $(\mathrm{CDI}-2-\mathrm{SF})$ is a 12 -item short-form, self-report measure of depression. ${ }^{21}$ Adequate internal consistency reliability was shown for the current sample $(\alpha=0.739)$. The Pediatric Quality of Life Inventory 4.0 (PedsQL; Generic Core Scales) is a 23-item measure of health-related QOL (HRQOL) developed by Dr James W. Varni that includes both self-report (completed by youth) and proxy-report (completed by caregivers) versions. ${ }^{22}$ The inventory has two domains: physical $(\alpha=0.815$ and 0.835 for self- and proxy-report versions, respectively) and psychosocial functioning ( $\alpha=0.834$ and 0.888 ). Psychosocial functioning is composed of three subscales: emotional $(\alpha=0.741$ and 0.811$)$, social $(\alpha=0.682$ and 0.773$)$ and school functioning $(\alpha=0.709$ and 0.810$)$, all of which demonstrated adequate internal consistency reliability with the current sample. Finally, the Children's Assessment of Participation and Enjoyment (CAPE. Copyright (C) 2004 NCS Pearson, Inc. Online adaptation copyright 2012 NCS Pearson, Inc. Adapted and reproduced with permission. All rights reserved) is a measure of participation in community activities, with subscales involving diversity (number of activities child engages in), intensity (amount of time of participation), with whom (whom child participates with) where (where child participates) and enjoyment. This measure has demonstrated adequate test-retest reliability and content and construct validity in the past research. ${ }^{23}$

\section{Data analyses}

This study used a mixed-methods approach. First, qualitative methods were used to analyze the written caregiver responses of youth (ages 1-18 years) to the open-ended questions. In particular, consistent with qualitative research methodology, inductive coding was incorporated, in that codes grew out of the data. The first author of this manuscript reviewed all caregiver responses and created a coding scheme to capture the range of responses. This coding scheme was then reviewed by the second and third authors in order to refine the categorizations based on caregiver responses, existing literature and our team's past research and clinical experience. Once the coding scheme was finalized, in order to facilitate the credibility of the results, ${ }^{24,25}$ investigator triangulation was incorporated, and all responses were coded by the first and second authors of this manuscript. Finally, disagreements were discussed until consensus was reached.

Second, we incorporated quantitative analyses to examine the relationships between caregiver-reported rewards and child, caregiver and community demographic characteristics. Further quantitative analyses were also conducted with a subsample of youth (ages 7-18 years) who had completed additional surveys (participation, anxiety, depression and HRQOL), with the purpose of assessing the relationships between caregiver-reported rewards and child psychosocial health. For quantitative analyses examining relationships between caregiver-reported rewards (dichotomous variables) and demographic characteristics, depending on whether the demographic variable was categorical or continuous, Chi square analyses were conducted or Point-biserial correlation coefficients were computed, respectively. ${ }^{26}$ As they are used to assess relationships between one dichotomous and one continuous variable, Point-biserial correlations were also computed to examine relationships between rewards and (continuous) psychosocial outcomes. Descriptive and inferential statistics were used to evaluate relationships between rewards of parenting and demographic characteristics of the child and caregiver. Specific to the qualitative findings, we have included direct quotes from participants in order to provide an accurate representation of caregiver perspectives. We have incorporated a similar methodology to address these types of data in the past research. ${ }^{27,28}$ Finally, we have included (anonymous) a participant number after each quote in order to demonstrate the representative nature of the quotes that were selected.

\section{RESULTS}

\section{Participants}

Two hundred and ninety-one primary caregivers participated in the larger, overall study, and 61\% $(n=178)$ responded to the open-ended 
Table 1 Characteristics

\begin{tabular}{|c|c|c|}
\hline Participant characteristics & $\begin{array}{c}\text { Caregivers who } \\
\text { answered the rewards } \\
\text { question }(n=178 \text { ) }\end{array}$ & $\begin{array}{c}\text { Caregivers of } \\
\text { youth aged } 7-18 \text { years } \\
\qquad(n=134)\end{array}$ \\
\hline & $\begin{array}{c}\text { Mean, median, } \\
\text { s.d., range }\end{array}$ & $\begin{array}{c}\text { Mean, median, } \\
\text { s.d., range }\end{array}$ \\
\hline $\begin{array}{l}\text { Demographics } \\
\text { Patient sex }\end{array}$ & $\%$ & $\%$ \\
\hline Male & 56 & 60 \\
\hline Female & 44 & 40 \\
\hline \multicolumn{3}{|l|}{ Patient race } \\
\hline Caucasian & 65 & 64 \\
\hline Latino & 17 & 18 \\
\hline African-American & 7 & 6 \\
\hline Asian & 3 & 3 \\
\hline Other & 8 & 9 \\
\hline Patient age at interview & $\begin{array}{c}11.07,12,5.23 \\
1-18\end{array}$ & $\begin{array}{c}13.43,14,3.53 \\
7-18\end{array}$ \\
\hline Patient age at injury & $\begin{array}{c}5.76,4,5.64 \\
0-17\end{array}$ & $\begin{array}{c}7.28,6,5.65 \\
0-17\end{array}$ \\
\hline \multicolumn{3}{|l|}{ Level of injury } \\
\hline Paraplegia & 68 & 66 \\
\hline Tetraplegia & 32 & 34 \\
\hline \multicolumn{3}{|l|}{ Extent of injury (American } \\
\hline \multicolumn{3}{|l|}{$\begin{array}{l}\text { Spinal Injury Association } \\
\text { Impairment Scale, AIS) }\end{array}$} \\
\hline AIS A (complete injury) & 49 & 49 \\
\hline $\begin{array}{l}\text { AIS B, C or D } \\
\text { (incomplete injury) }\end{array}$ & 51 & 51 \\
\hline \multicolumn{3}{|l|}{ Caregiver education } \\
\hline No college & 26 & 26 \\
\hline $\begin{array}{l}\text { At least some level of } \\
\text { college }\end{array}$ & 74 & 74 \\
\hline \multicolumn{3}{|l|}{ Caregiver relation to patient } \\
\hline Mother & 82 & 80 \\
\hline Father & 12 & 12 \\
\hline Grandmother & 3 & 4 \\
\hline Other & 2 & 3 \\
\hline Stepmother & 1 & 1 \\
\hline \multicolumn{3}{|l|}{ Type of community } \\
\hline Rural/small town & 49 & 50 \\
\hline Urban/city & 51 & 50 \\
\hline
\end{tabular}

question regarding the rewards they get from parenting their child so were included in the current study. Analyses revealed that the caregivers who did and did not respond to the reward question did not differ in terms of their sex $\chi^{2}(1, N=150)=0.30, P=0.59$, type of community $\chi^{2}(1, N=201)=0.71, P=0.40$ or whether or not they were employed $\chi^{2}(1, N=200)=0.39, P=0.54$, but responders were more likely to have some college experience $\chi^{2}(1, N=202)=6.52$, $P=0.01$, and more likely to be married $\chi^{2}(1, N=200)=4.76, P=0.03$. For the 178 participating primary caregivers and their children with
Table 2 Caregiver-reported rewards of parenting youth with $\mathrm{SCI}$

\begin{tabular}{lc} 
Rewards & $\begin{array}{c}\text { Percentage } \\
\text { endorsed (\%) }\end{array}$ \\
\hline $\begin{array}{lc}\text { Enhanced Resilience } \\
\text { Resilience in my Child (for example, child works really hard, }\end{array}$ & 71.90 \\
overcomes obstacles, improves, has a positive attitude) & \\
Resilience in Myself (for example, finding strength/growth, & 19.10 \\
appreciating what we have, hope for the future) & \\
& 18 \\
Caregiver-Child Relationships & 11.20 \\
Connecting with Others & 9.60 \\
Learning & 2.20 \\
No Rewards & 3.40 \\
Same as Parenting any Other Child & \\
\hline
\end{tabular}

Abbreviation: $\mathrm{SCl}$, spinal cord injury.

Percentage of entire sample that endorsed each reward category/subcategory. Many caregivers endorsed multiple rewards, so percentages do not sum to $100 \%$.

SCI, youth were between 1 and 18 years of age at the time of interview $($ mean $=11.06$ years, s.d. $=5.22), 56 \%$ were male, $68 \%$ had paraplegia and, on average, they were injured at age 5.76 (s.d. $=5.63$ ) years. Most of the caregivers were mothers $(82 \%)$ and had at least some college experience $(74 \%)$. Fifty-one percent of the families lived in an urban area.

As mentioned above, caregivers of youth aged $7-18$ years $(n=134)$ were included in subsequent analyses to explore relationships between caregiver-reported rewards and child psychosocial outcomes. Aside from age-related variables, there were no significant differences in terms of demographic characteristics between the 134 youth/caregivers who were aged 7-18 years and the remaining 44 youth/caregivers who were aged 1-6 years. Demographics for both groups (that is, all 178 caregivers who answered the rewards question and the 134 caregivers who answered the rewards question and have children aged 7-18 years) are presented in Table 1 .

\section{Describing caregiver-reported rewards}

Results indicate that caregivers derive a variety of rewards from parenting their children with SCI (see Table 2). In particular, we categorized caregiver-reported rewards as including Enhanced Resilience (mentioned by $71.9 \%$ of caregivers), a stronger Caregiver-Child Relationship (mentioned by $18 \%$ of caregivers), Connecting with Others (mentioned by $11.2 \%$ of caregivers) and Learning (mentioned by $9.6 \%$ of caregivers). Finally, $3.4 \%$ of caregivers reported that parenting a child with SCI was like parenting any other child, and $2.2 \%$ reported that there were no rewards to parenting their child with SCI. Important to note, some caregivers mentioned more than one reward, so percentages do not add up to 100. As mentioned above, anonymous participant number is presented with each quote to represent the range of responses across participating caregivers.

Enhanced Resilience emerged from the analyses as an important caregiver-reported reward as it was endorsed most frequently (71.9\%) out of the four categories. With several subcategories and subthemes, this category also included the most codes. The subcategory Resilience in my Child captured caregivers referring to their child's accomplishments, improvement/recovery, school/academic achievements and having a positive/optimistic attitude. One caregiver wrote, 'He makes a lot of people smile because of his outgoing attitude. Seeing others smile is very rewarding' (participant no. 131). The subcategory Resilience in Myself captured caregivers referring to appreciating what 
they have, finding strength/growth and learning new ways of living and being. 'I previously would have felt sorry for someone dealing with his issues', wrote a caregiver. 'Now I see it as life and am very thankful for what we DO have instead of sad over what is lost' (no. 159). Finally, the subcategory Resilience in my Family captured rewards related to the unconditional love of their family members (including their other children) toward their child with SCI. 'We as a family have become stronger and closer since his injury', wrote a caregiver (no. 262). Although Enhanced Resilience is being presented here as one coherent theme, subsequent preliminary analyses identified different patterns between those who reported 'Resilience in Myself and 'Resilience in my Child'. Therefore, subsequent analyses were conducted using these subcategories rather than the overall category of Enhanced Resilience.

Eighteen percent of caregivers reported having a closer CaregiverChild Relationship. Example codes in this category included spending time together, growing close and providing child with emotional assistance. One caregiver wrote that it was rewarding just being her mom. We are closer because we have had to spend a lot of time together that moms and typical kids don't have to' (no. 12). Another caregiver described her reward as, 'helping him recover so fully and being able to be with him and help him' (no. 116). Interestingly, one caregiver noted that: 'Our relationship is closer (though sometimes it's too close)' (no. 271).

Just over $11 \%$ of caregivers endorsed Connecting with Others. Subcategories emerged from the data, such as Building Relationships, which captured themes including building relationships with other children and families, with providers and with organizations. One caregiver felt rewarded by 'meeting all the exceptional people and other families who have gone through similar experiences' (no. 132). The subcategory Receiving Support captured themes including receiving support from family/friends, community, health-care providers/therapists and spiritual guidance. A caregiver commented on how helpful it was to discover 'so much empathy from the community' (no. 9). Spirituality enabled some caregivers to let go of aspects of their child's life that they or their child could not change. A caregiver wrote, 'Knowing that God was with us every step of the way from injury to amazing testimony' (no. 218). Finally, Giving Support to Others Besides the Child incorporated the notion that caregivers were rewarded by their involvement in different organizations and educating others about SCI. A caregiver wrote about how rewarding it was 'working with organizations that assist families and kids with disabilities' (no. 89).

Nearly $10 \%$ of caregivers reported several aspects of Learning as a reward, including learning about new experiences, learning about SCI, learning about the child, learning from the child, the caregiver and child learning from each other and learning to advocate. A caregiver wrote that learning about the injury was so rewarding because it taught her 'all the new stuff to be able to take care of her child' (no. 69). Another caregiver wrote, 'Learning what he can learn to do for himself, and seeing what he is capable of (no. 183). Finally, caregivers wrote about learning to advocate for their children as an opportunity for growth.

Just over 3\% of caregivers reported that the rewards of parenting their child with SCI were no different than parenting a child without SCI. For example, a caregiver wrote, 'Rewards in regard to parenting are no different than parenting any child-but my child is very high functioning' (no. 42). Specific to the $2.2 \%$ of caregivers who cited no rewards, one caregiver wrote 'not sure there is anything I would consider rewarding about it' (no. 109). Another caregiver responded, 'I love my child, but do not find her condition rewarding. Have not been able to reconcile situation' (no. 209). Finally, a caregiver mentioned that the rewards of parenting come in stages. 'At this stage, I am trying to understand and accept the changes, work with my child. I want to help him to adapt to his life. Maybe later I will see things in a different way' (no. 287). Because of the low frequency of endorsement, these last two categories (that is, rewards are same as parenting any other child and not seeing any rewards) were included here for descriptive purposes only and were not included in subsequent analyses.

\section{Relationships between caregiver-reported rewards and demographic characteristics}

We next examined the relationships between caregiver-reported rewards and demographic characteristics among all 178 respondents. Specifically, quantitative analyses were conducted to identify relationships between caregiver-reported rewards and child sex, current age, age at injury, level of injury and type of community and caregiver marital status, education and employment.

In terms of findings, caregivers who had children who were older at the time of injury $(r=0.268, n=177, P<0.001)$ and interview $(r=0.179, n=178, P=0.017)$ were more likely to report having an enhanced Caregiver-Child Relationship. Caregivers without college exposure $\left(\chi^{2}(1, N=171)=4.49, P=0.034\right)$ and those who were unemployed $\left(\chi^{2}(1, N=169)=4.77, P=0.029\right)$ were more likely to cite Learning as a reward. No other significant relationships emerged.

\section{Relationships between caregiver-reported rewards and youth psychosocial outcomes}

As mentioned above, youth aged $7-18$ years $(n=134)$ completed surveys assessing their participation, anxiety, depression and HRQOL, and their caregivers completed a survey assessing their children's HRQOL. Significant relationships emerged between caregiver-reported rewards and child participation, HRQOL (child self-report and caregiver proxy report) and depression (see Table 3). In particular, caregivers whose children scored lower on self-reported school and psychosocial HRQOL were more likely to report 'Resilience in my Child' as a reward. Caregivers whose children participated in fewer community activities and participated less often and had lower levels of depressive symptoms reported experiencing an Enhanced Caregiver-Child Relationship. Finally, caregivers whose children participated in activities further from home and with a broader group and had higher self-reported psychosocial HRQOL and self-reported and parent-reported school HRQOL were more likely to report Connecting with Others as a reward. No other significant relationships emerged.

\section{DISCUSSION}

Most of the caregiver literature has focused on the negative impacts of caregiving, which has provided valuable information on the needs of caregivers and how interventions might meet those needs. However, there is a dearth of literature regarding the positive experiences of caregivers. As mentioned, previous research has underlined a variety of factors associated with resilience. Resilience has been described as the process in which an individual positively adapts to negative psychological sequelae. Understanding these resilient experiences may help identify protective factors that can be incorporated by others in the face of adversity. The current study aimed to describe the positive experiences reported by caregivers related to parenting their children with SCI and explore how these rewards relate to demographic characteristics and youth psychosocial outcomes. 
Table 3 Correlations between caregiver-reported rewards and youth psychosocial outcomes

\begin{tabular}{|c|c|c|c|c|c|}
\hline & \multicolumn{2}{|c|}{ Enhanced Resilience } & \multirow{3}{*}{$\begin{array}{c}\text { Caregiver-Child Relationship } \\
r\end{array}$} & \multirow{3}{*}{$\begin{array}{c}\text { Connecting with Others } \\
\text { r }\end{array}$} & \multirow{3}{*}{$\frac{\text { Learning }}{r}$} \\
\hline & Resilience in my Child & Resilience in Myself & & & \\
\hline & r & $r$ & & & \\
\hline \multicolumn{6}{|l|}{ Rewards } \\
\hline Intensity & 0.068 & -0.017 & $-0.191^{*}$ & -0.049 & 0.063 \\
\hline With whom & -0.026 & -0.084 & -0.027 & $0.195^{*}$ & -0.135 \\
\hline Where & 0.104 & -0.092 & -0.106 & $0.225^{*}$ & -0.169 \\
\hline Enjoyment & -0.074 & -0.038 & 0.027 & -0.178 & 0.117 \\
\hline \multicolumn{6}{|c|}{ Child HRQOL subdomains } \\
\hline Psychosocial & $-0.214^{*}$ & -0.002 & 0.098 & $0.207^{*}$ & -0.008 \\
\hline \multicolumn{6}{|c|}{ Caregiver proxy on Child HRQOL subdomains } \\
\hline Physical & -0.1 & -0.05 & 0.019 & 0.092 & 0.015 \\
\hline Emotional & -0.031 & 0.07 & 0.02 & 0.144 & -0.104 \\
\hline Social & -0.059 & -0.048 & 0.026 & 0.009 & 0.005 \\
\hline School & -0.081 & -0.01 & 0.03 & $0.236^{* *}$ & -0.025 \\
\hline Psychosocial & -0.064 & 0.013 & 0.02 & 0.153 & -0.045 \\
\hline Child anxiety & 0.111 & -0.054 & -0.124 & -0.131 & -0.027 \\
\hline Child depression & 0.015 & -0.091 & $-0.201^{*}$ & -0.032 & -0.063 \\
\hline
\end{tabular}

Abbreviation: HRQOL, health-related quality of life.

${ }^{*} P<0.05,{ }^{* *} P<0.01$.

Results provided valuable perspectives on the rewards of parenting a child with SCI. In particular, four overarching categories of rewards emerged, in that caregivers reported experiencing enhanced: Resilience, Caregiver-Child Relationships, Connections with Others and Learning. Previous studies have not looked at these four caregiver rewards in the context of pediatric SCI. However, we know that children who face chronic adversity recover better when they have a positive relationship with an adult, when they interact with others, feel self-confident and have a sense of belonging in the context of the community. ${ }^{29,30}$ We also know that caregivers of youth are in a unique position to draw on internal and external resources that can affect parent and child well-being. ${ }^{31}$ In our study, the vast majority of caregivers of youth with SCI responded to a question about the rewards of caregiving by focusing on the positive aspects of parenting such as their child's accomplishments and their close parent-child relationship. Additionally, caregivers emphasized their own achievements and strengths. In particular, caregivers in our study talked about building social networks, receiving support from the community and gathering information from health-care professionals. A better understanding of how caregivers use these resources to transcend difficult challenges would be valuable to future intervention development and is a point for further research.

Results revealed relationships between demographics and the themes Caregiver-Child Relationship and Learning. In particular, caregivers who felt rewarded by having an enhanced relationship with their child were more likely to have children who were older, both currently and at the time of injury. This could represent a reconnecting of adolescents with SCI with their caregivers after their injury. Interestingly, one caregiver wrote that their caregiver-child relationship was too close. Past research has indicated the increased importance of families to youth with disabilities because they may be more likely to experience social isolation from peers. ${ }^{32}$ This falls in contrast to typical adolescent development, where peer connections often become the primary relationships for youth, surpassing parent influence. Perhaps this parent noticed an increased closeness with their child (as opposed to stronger relationships between their child and his/her peers) as not necessarily in line with typical development.

Specific to learning, caregivers who had no college exposure and those who were unemployed reported benefiting from learning after their child's injury, including learning more about their children and themselves. This may indicate a particular need and appreciation for further information among those with less formal education and less access to networks of support that may be provided through employment. Moreover, this may further emphasize the need by those with less formal education to have access to a wide range of learning resources, educational tools and comprehensive services early in the rehabilitation process to help them best care for their children. Future research should attend to the needs of these caregivers as they may be at risk for particular marginalization after their child's injury.

The present study also identified relationships between caregiverreported rewards and child psychosocial outcomes. Relationships were found between child psychosocial outcomes and the subcategory 'Resilience in my Child' and the themes Caregiver-Child Relationship and Connecting with Others. In particular, parents whose children 
had lower levels of child-reported school and psychosocial HRQOL reported seeing 'Resilience in my Child' as a reward of caregiving. Although this may seem paradoxical, it may be that, for youth who are struggling with school-related challenges, their parents are more likely to identify their struggle and acknowledge their perseverance despite the obstacles they face. Toward this end, it may be that lower school and psychosocial HRQOL are driving caregivers to acknowledge the resilience in their children. Further research should continue to investigate this relationship.

A significant relationship was also found between caregivers citing the Caregiver-Child Relationship as a reward and youth's community participation and mental health. Specifically, caregivers of children who participated in fewer activities and participated less often, but also had less depressive symptoms were more likely to mention their relationship with their child as a caregiving reward. As our past research has found older youth to participate in fewer activities, ${ }^{33}$ it is possible that the relationship between community participation and an enhanced caregiver-child relationship is an artifact of the finding reported above regarding child age. Or perhaps a closer caregiverchild relationship precludes youth from spending time engaged in community activities or vice versa. One study found that parents of children with diabetes were more likely to be overprotective, particularly for younger children, where parents felt the need to significantly manage their child's diabetes regimen. ${ }^{34}$ The connection between caregiver-child relationship and decreased depressive symptomatology among youth may support previous findings that the parent-child relationship may serve as a protective factor against youth depression. ${ }^{35}$ However, it is important to point out that, while past research has shown a reciprocal relationship between child and caregiver mental health, ${ }^{36}$ future longitudinal research should strive to understand the direction of all of these relationships. The importance of the caregiver-child relationship, particularly as the child moves through various developmental stages, warrants attention in future research.

Finally, caregivers who had children reporting higher levels of selfreport psychosocial HRQOL and (self-report and parent report) school HRQOL were more likely to feel rewarded by Connecting with Others. Consistent with the literature, social support is associated with higher levels of adaptability and adjustment while social isolation has been linked to higher levels of anxiety and depression. ${ }^{37,38,39}$ Social support may also help caregivers and youth expand their social networks, which may provide resources and strategies that are helpful throughout the caregiving process. Future interventions should continue to strengthen natural supports in the community that can help caregivers and youth cope throughout the process of adjustment to (and life with) SCI.

Although notable, it was reassuring that only a few caregivers reported gaining no rewards from parenting their child with SCI. Yet, these statements importantly reflect the reality of the difficult nature of SCI and of going through the process of watching your child experience a traumatic injury. Additionally, a few caregivers reported that parenting a child with SCI was the same as parenting any other child. These statements highlight the notion that a disability is only one characteristic of a child and that parenting any child, regardless of that child's ability, has challenges and rewards. Underscoring the significance of these statements is the notion of providing support to caregivers immediately after injury, during rehabilitation and after discharge as they continue caring for their growing child.

\section{Limitations}

There were several limitations to the current study. First, these data are cross-sectional (and analyses were correlational); therefore, no causal inferences can be drawn from this study. Future longitudinal research would be useful in understanding the connections between caregiver and youth functioning. Second, the group of caregivers who responded to the rewards question differed from the overall sample in that they were more likely to have had some college education and they were more likely to be married. It could be that those with more resources in terms of education and (presumably) social support either felt more comfortable in answering the question or were better able to identify caregiving rewards. This is an area for future research, particularly as we strive to understand the experiences of the diverse group of families who experience SCI. Third, the majority of the primary caregivers in this study were mothers, and as a result, participants included only a small number of fathers and other caregivers. Although finding the majority of primary caregivers to be mothers is consistent with our past research, ${ }^{40}$ future work should strive to understand the perspectives of fathers and other caregivers and how their experiences may relate to the health and well-being of their children. Additionally, methodological limitations included recruiting from only one specialty hospital system in the United States as well as the self-report nature of our data, which may hinder our ability to generalize these findings to the general population of caregivers and youth with SCI. Further, while asking caregivers an open-ended question about rewards allowed for the collection of a wide range of types of rewards, because the qualitative data in this study were written and not collected as part of an interview, any uncertainty about the content or intent of the answers could not be clarified by the caregivers themselves. Finally, owing to the lack of systematic ways to assess self-report data on health and functioning from youth aged $<7$ years, we were unable to examine how caregiverreported rewards related to the adjustment of younger children.

\section{CONCLUSION}

This mixed-method study examined caregiver-reported rewards of parenting youth with SCI and found rewards to include four themes: Enhanced Resilience, an Enhanced Caregiver-Child Relationship, Connecting with Others, and Learning. Analyses revealed significant relationships between caregiver-reported rewards and demographic characteristics as well as youth psychosocial outcomes, indicating that positive adjustment of caregivers is an important area of future research. In particular, future research should examine whether these four emerging themes can be fostered as factors that protect caregivers from distress and potentially yield more positive long-term outcomes for their children with SCI.

\section{DATA ARCHIVING}

There were no data to deposit.

\section{CONFLICT OF INTEREST}

The authors declare no conflict of interest.

\section{ACKNOWLEDGEMENTS}

We thank the project staff, all youth and caregivers who participated in this project and Shriners Hospitals for Children (Grant no. 79143) for funding this study. 
1 Perrin JM. Health services research for children with disabilities. Milbank Q 2002; 80: 303-324.

2 National Institute of Neurological Disorders and Stroke. [Internet] 2014 (cited 31 July 2014); Available from http://www.ninds.nih.gov/disorders/sci/sci.htm.

3 Klaas S, Kelly E, Anderson C, Vogel L. Depression and anxiety in adolescents with pediatric-onset spinal cord injury. Top Spinal Cord Inj Rehabil 2014; 20: 13-22.

4 Kerstin W, Gabriele B, Richard L. What promotes physical activity after spinal cord injury? An interview study from a patient perspective. Disabil Rehabil 2006; 28: 481-488.

5 Johnson KA, Klaas SJ, Vogel LC, McDonald C. Leisure characteristics of the pediatric spinal cord injury population. J Spinal Cord Med 2003; 27: S107-S109.

6 Hammell KW. Experience of rehabilitation following spinal cord injury: a meta-synthesis of qualitative findings. Spinal Cord 2007; 45: 260-274.

7 Witt WP, Riley AW, Coiro MJ. Childhood functional status, family stressors, and psychosocial adjustment among school-aged children with disabilities in the United States. Arch Pediatr Adolesc Med 2003; 157: 687-695.

8 Vermaes IP, Janssens JM, Bosman AM, Gerris JR. Parents' psychological adjustment in families of children with spina bifida: a meta-analysis. BMC Pediatrics 2005; 5: 1.

9 Garma SI, Kelly EH, Daharsh EZ, Vogel LC. Health-related quality of life after pediatric spinal cord injury. J Pediatr Psychol 2011; 36: 226-236.

10 Raina P, O'Donnell M, Rosenbaum P, Brehaut J, Walter SD, Russell D et al. The health and well-being of caregivers of children with cerebral palsy. Pediatrics 2005; 115: e626-e636.

11 Kuo DZ, Cohen E, Agrawal R, Berry JG, Casey PH. A national profile of caregiver challenges among more medically complex children with special health care needs. Arch Pediatr Adolesc Med 2011; 165: 1020-1026.

12 Tugade MM, Fredrickson BL. Resilient individuals use positive emotions to bounce back from negative emotional experiences. J Pers Soc Psychol 2004: 86: 320.

13 Pinquart M, Sörensen S. Associations of stressors and uplifts of caregiving with caregiver burden and depressive mood: a meta-analysis. J Gerontol B Psychol Sci Soc Sci 2003; 58: P112-P128.

14 Ruiz-Robledillo N, De Andrés-García S, Pérez-Blasco J, González-Bono E, Moya-Albiol L. Highly resilient coping entails better perceived health, high social support and low morning cortisol levels in parents of children with autism spectrum disorder. Res Dev Disabil 2014; 35: 686-695.

15 Simpson G, Jones K. How important is resilience among family members supporting relatives with traumatic brain injury or spinal cord injury? Clin Rehabil 2012; 27: 367-377.

16 Woodman AC, Hauser-Cram P. The role of coping strategies in predicting change in parenting efficacy and depressive symptoms among mothers of adolescents with developmental disabilities. J Intellect Disabil Res 2013; 57: 513-530.

17 Belsky J. The determinants of parenting: a process model. Child Dev 1984; 55: 83-96.

18 Masten AS, Best KM, Garmezy N. Resilience and development: contributions from the study of children who overcome adversity. Dev Psychopathol 1990; 2: 425-444.

19 Kirshblum SC, Burns SP, Biering-Sorensen F, Donovan W, Graves DE, Jha A et al. International standards for neurological classification of spinal cord injury (revised 2011). J Spinal Cord Med 2011; 34: 535-546.

20 Reynolds CR, Richmond BO. Revised Children's Manifest Anxiety Scale. Western Psychological Services: Los Angeles, CA, USA, 1985.

21 Kovacs M, Staff M. Children's Depression Inventory (CDI): Technical Manual Update. Multi-Health Systems. Inc.: North Tonawanda, NY, USA, 2003.
22 Varni JW, Seid M, Kurtin PS. PedsQL 4.0: reliability and validity of the Pediatric Quality of Life Inventory Version 4.0 Generic Core Scales in healthy and patient populations. Med Care 2001; 39: 800-812.

23 King G, Law M, King S, Hurley P, Hanna S, Kertoy M et al. Children's Assessment of Participation and Enjoyment (CAPE) and Preferences for Activities of Children (PAC). Harcourt Assessment: San Antonio, TX, USA, 2004.

24 Gorzkowski J, Kelly EH, Klaas SJ, Vogel LC. Obstacles to community participation among youth with spinal cord injury. J Spinal Cord Med 2011; 34: 576-585.

25 House LA, Russell HF, Kelly EH, Gerson A, Vogel LC. Rehabilitation and future participation of youth following spinal cord injury: caregiver perspectives. Spinal Cord 2009; 47: 882-886.

26 Lincoln YS, Guba EG. Naturalistic Inquiry, Sage Publications, Ltd: Newbury Park, CA, USA, 1985.

27 Patton MQ. Qualitative Research \& Evaluation Methods 3rd edn. Sage Publications: Thousand Oaks, CA, USA, 2002.

28 Pett MA. Nonparametric Statistics for Health Care Research. Sage Publications Inc.: Thousand Oaks, CA, USA, 1997, pp 312.

29 Kaplan CA, Owens J. Parental influences on vulnerability and resilience. In: Hoghughi M, Long N (eds). Handbook of Parenting. Theory and Research Practice, vol. 8. Sage: London, UK, 2004, pp 55-71.

30 Masten AS, Best KM, Garmezy N. Resilience and development: contributions from the study of children who overcome adversity. Dev Psychopathol 1990; 2: 425-444.

31 Sapienza JK, Masten AS. Understanding and promoting resilience in children and youth. Curr Opin Psychiatry 2011; 24: 267-273.

32 Holmbeck GN, Westhoven VC, Phillips WS, Bowers R, Gruse C, Nikolopoulos T et al. A multimethod, multi-informant, and multidimensional perspective on psychosocial adjustment in preadolescents with spina bifida. J Consult Clin Psychol 2003; 71: 782.

33 Klaas SJ, Kelly EH, Gorzkowski J, Homko E, Vogel LC. Assessing patterns of participation and enjoyment in children with spinal cord injury. Dev Med Child Neurol 2010; 52: 468-474.

34 Mullins LL, Fuemmeler BF, Hoff A, Chaney JM, Van Pelt J, Ewing CA. The relationship of parental overprotection and perceived child vulnerability to depressive symptomotology in children with type 1 diabetes mellitus: the moderating influence of parenting stress. Children's Health Care 2004; 33: 21-34.

35 Raja SN, McGee R, Stanton WR. Perceived attachments to parents and peers and psychological well-being in adolescence. J Youth Adolesc 1992; 21: 471-485.

36 Boyer BA, Knolls ML, Kafkalas CM, Tollen LG, Swartz M. Prevalence and relationships of posttraumatic stress in families experiencing pediatric spinal cord injury. Rehabil Psychol 2000; 45: 339.

37 La Greca AM, Bearman KJ, Moore H. Peer relations of youth with pediatric conditions and health risks: promoting social support and healthy lifestyles. J Dev Behav Pediatr 2002; 23: 271-280.

38 Beedie A, Kennedy P. Quality of social support predicts hopelessness and depression post spinal cord injury. J Clin Psychol Med Settings 2002; 9: 227-234.

39 Dumont M, Provost MA. Resilience in adolescents: Protective role of social support, coping strategies, self-esteem, and social activities on experience of stress and depression. J Youth Adolesc 1999; 28: 343-363.

40 Kelly EH, Anderson CJ, Garma SI, Russell HF, Klaas SJ, Gorzkowski JA et al. Relationships between the psychological characteristics of youth with spinal cord injury and their primary caregivers. Spinal Cord 2011; 49: 200-205. 